\title{
Automatic Repair of Same-Timestamp Errors in Business Process Event Logs
}

\author{
Raffaele Conforti ${ }^{1, \star}$, Marcello La Rosa ${ }^{2}$, Arthur H.M. ter Hofstede ${ }^{3}$, and \\ Adriano Augusto ${ }^{2}$ \\ 1 Process Diamond, Australia \\ rconforti@processdiamond.com \\ 2 The University of Melbourne, Australia \\ \{marcello.larosa, a.augusto\}@unimelb.edu.au \\ 3 Queensland University of Technology, Australia \\ a.terhofstede@qut.edu.au
}

\begin{abstract}
This paper contributes an approach for automatically correcting "same-timestamp" errors in business process event logs. These errors consist in multiple events exhibiting the same timestamp within a given process instance. Such errors are common in practice and can be due to the logging granularity or the performance load of the logging system. Analyzing logs that have not been properly screened for such problems is likely to lead to wrong or misleading process insights. The proposed approach revolves around two techniques: one to reorder events with same-timestamp errors, the other to assign an estimated timestamp to each such event. The approach has been implemented in a software prototype and extensively evaluated in different settings, using both artificial and real-life logs. The experiments show that the approach significantly reduces the number of inaccurate timestamps, while the reordering of events scales well to large and complex datasets. The evaluation is complemented by a case study in the meat $\&$ livestock domain showing the usefulness of the approach in practice.
\end{abstract}

\section{Introduction}

In real-life scenarios, one regularly finds inaccurate or unreliable data in the records of business process executions (known as event logs) [1,2]. A common case of inaccurate data in event logs regards the recording of timestamps. Each event in an event log captures the start or completion of a process activity and as such has a timestamp, e.g. activity "Reject purchase order" was completed at 12:30:00pm of 23/02/2020. A common type of timestamp error is when a set of events related to the same process instance have the same timestamp. This error can be due to delays during the logging process, e.g. when the logging system becomes overloaded; or to the granularity of the logging, e.g. the events do not capture the time but only the date. This is a frequent issue when the logging is done manually [3] or when the logging system is a legacy system.

Analyzing event logs that have not been screened for timestamp errors is likely to lead to wrong or misleading insights. For example, in the context of automated process

\footnotetext{
* Work done while the author was at The University of Melbourne and Queensland University of Technology.
} 
discovery, where the objective is to automatically discover a process model from an event $\log$, the presence of such errors may lead to mixing up order dependencies between process activities, especially if the logging system does not guarantee that events are recorded in the order of completion of the respective activities, or if the logging system may collect data from different sources. For example, given two activities A and B with same timestamp, these may erroneously be assumed to be concurrent, i.e. they can be executed in any order in the resulting process model, while in reality A is causal to B, i.e. A must always precede B in any instance of the process model. Any insights derived from a process model whose activities have wrong order dependencies, will be misleading. Timestamp errors can also affect performance statistics derived from the event $\log$ such as activity durations and waiting times. These statistics are used in the context of performance mining, variant analysis and conformance checking techniques, for example when checking the conformance of the as-is process to business rules that involve temporal aspects of the process. Thus, timestamp errors may also lead to wrong insights on the performance or conformance of the process at hand.

While many approaches have been proposed to filter out or repair events in order to improve the overall quality of an event $\log [4-12]$, only two of them $[6,8]$ address the problem of repairing timestamp errors and they do so under a strict requirement: the presence of a (reference) process model with execution time annotations as input.

In this paper, we propose an automated approach to repairing timestamp errors in event logs. Specifically, we address the case where multiple events belonging to the same process instance (called trace) have the same timestamp. Our approach to tackle the "same-timestamp" error relies on two techniques. The first technique estimates, for each trace, the most-likely order between the events affected by the same-timestamp error, using information from correctly-ordered events in the log, and repairs the log accordingly. The second technique assigns a timestamp to each event that has been reordered, based on an estimation of the duration of each process activity in the log.

We implemented our approach in a software prototype and conducted a series of experiments with artificial and real-life logs, aimed at measuring the accuracy and time performance of our approach in correcting same-timestamp errors, under different settings. In addition, we carried out a case study with an Australian organization in the meat $\&$ livestock domain as an initial assessment of the usefulness of our approach in practice.

This paper is organized as follows. Section 2 introduces the required background and discusses approaches for event log filtering and repair. Section 3 presents our approach. Section 4 reports the experimental evaluation, including our case study. Section 5 concludes the paper and discusses future work.

\section{Background and Related Work}

Organizations often keep records of the execution of their business processes, e.g. orderto-cash or procure-to-pay processes, in the form of event logs. An event log is a set of traces where each trace captures the execution of a particular process instance (a.k.a. case). Traces are recorded as sequences of events and are identified by a unique case identifier (case id). Each event refers to the execution of a specific activity within a 
process case at a specific time. For example, the event with an activity labeled "Invoice released", a case id "134", and timestamp "23-02-2020T12:30:00", indicates that an invoice has been released for case 134 at 12:30 of 23 February 2020.

Process mining aims at automatically extracting actionable process knowledge from event logs $[13,14]$. This knowledge may take the form of a process model, a process performance report, and other artifacts. Data pre-processing is that phase of a process mining project where raw data is transformed into an understandable format, so that it can be used as input for knowledge discovery techniques. A key step of data preprocessing is data cleaning, which includes: (i) the removal of outlier data, and (ii) the correction of erroneous data. When the data under consideration is an event log, the two activities are known as log filtering and log repair.

Log filtering is a de-facto practice performed before starting any type of process mining analysis. Filtering a log generally follows an unstructured approach as different logs may be affected by different issues. Previous studies $[2,1]$ have identified a collection of recurrent quality issues that commonly affect event logs, among these issues also incorrect timestamps. However, in the literature, log filtering is usually addressed from a noise-oriented perspective, focusing on removing infrequent traces and/or events from an event $\log [4,5,9]$ or event stream $[11,12]$.

The goal of log repair is to detect erroneous or missing data (e.g. an event or its attributes) and repair it by relying on a reference model or observed correct data. RoggeSolti et al. [6] propose to identify and restore missing events using a reference stochastic Petri net annotated with execution times. The method aligns each trace in the log to the model and, if the alignment is not perfect, the trace is repaired using the minimum cost alignment. Then, each event added as a result of the repair is assigned a timestamp computed via Bayesian networks. Similarly, Wang et al. [7] design an approach that, given a causal net, detects the events having an incorrect activity label and uses the net to restore the correct activity label. The main difference with [6] is that Wang et al. do not add new events to the log, but only repair the erroneous ones. Complementing the work of Wang et al., Shaoxu et al. [8] propose an approach to repair missing and incorrect timestamps, e.g. timestamps capturing only the hours. This approach determines the most likely correct timestamps with the aid of input time constraints between the observation of two consecutive activities. The new timestamps are assigned with the goal of minimizing the distance from input time constraints. Finally, Sani et al. [10] propose a general repair approach for infrequent behavior. Their main idea is to identify pairs of subtraces that are observed frequently (in different traces) having a third (variable) subtrace in between. Once the pairs are identified, the occurrences of the inner subtraces are analyzed to detect the least frequent ones, which are then replaced by frequent ones.

\section{Approach}

In this section, we present our approach for repairing event logs that contain traces where multiple events have the same timestamp and may, as such, be incorrectly ordered. We start by formally introducing the required preliminary concepts. Then, we describe our approach to reorder timestamp-equivalent events; we analyse its complex- 
ity and show its ILP formulation. Finally, we discuss a complementary technique to assign a timestamp to each reordered event.

\subsection{Preliminaries}

We define the notion of event log and the strictly-before and directly-follows relations between events. Using these, we provide the notion of log automaton.

Definition 1. [Event Log] Let $\Gamma$ be a finite set of activities. A $\log \mathcal{L}$ over $\Gamma$ is defined as $\mathcal{L} \triangleq(\mathcal{E}, \mathcal{C}, \mathcal{A}, \mathcal{T},<)$ where $\mathcal{E}$ is the set of events, $\mathcal{C}: \mathcal{E} \rightarrow \mathbb{N}$ is a surjective function linking events to cases, $\mathcal{A}: \mathcal{E} \rightarrow \Gamma$ is a function linking events to activities, $\mathcal{T}: \mathcal{E} \rightarrow \mathbb{N}$ is a function linking events to timestamps, and $\langle\subseteq \mathcal{E} \times \mathcal{E}$ is a strict total ordering over the events based on the sequential position of the events (as recorded in the event log and not according to their timestamp).

Definition 2. [Strictly-Before Relation] Given a $\log \mathcal{L}$ and two events $e_{1}, e_{2} \in \mathcal{E}$, e $e_{1}$ comes strictly-before $e_{2}$, i.e. $e_{1} \sqsubset e_{2}$, iff $e_{1}<e_{2} \wedge \mathcal{C}\left(e_{1}\right)=\mathcal{C}\left(e_{2}\right) \wedge \nexists e_{3} \in \mathcal{E} \mid \mathcal{C}\left(e_{3}\right)=\mathcal{C}\left(e_{1}\right) \wedge e_{1}<$ $e_{3} \wedge e_{3}<e_{2}$.

Definition 3. [Directly-Follows Relation] Given a $\log \mathcal{L}$ and two activities $x, y \in \Gamma, y$ directly follows $x$, i.e. $x \rightsquigarrow y$, iff $\exists e_{1}, e_{2} \in \mathcal{E} \mid \mathcal{A}\left(e_{1}\right)=x \wedge \mathcal{A}\left(e_{2}\right)=y \wedge e_{1} \sqsubset e_{2}$. Additionally, we introduce the function \# : $\Gamma \times \Gamma \rightarrow \mathbb{N}$ which retrieves the number of times a directly-follows relation occurs in the underlying log.

The directly-follows relations recorded in a $\log$ can be summarised with a directed graph, where each node represents an activity, and each arc connecting two nodes represents a directly-follows relation between the corresponding activities. From here on, we refer to this graph as the log automaton (L-automaton).

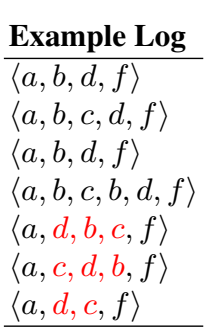

Table 1 Depending on the log quality, the L-automaton may contain several infrequent directly-follows relations. In fact, filtering the $\log$ or its L-automaton is common in many process discovery techniques $[15,16]$. To improve the quality of the Lautomaton, and the effectiveness of our technique, we recommend to apply one of the many techniques to remove infrequent behavior from the $\log [4,5,9]$. In this paper, we rely on the method in [5]. We refer to the filtered L-automaton as the filtered log automaton (F-automaton). By construction, the Fautomaton's arc set is a subset of the L-automaton's arc set. Figures $1 \mathrm{a}$ and $1 \mathrm{~b}$ show the L-automaton and the F-automaton of the log in Table 1.

\subsection{Repairing Events Order}

Starting from the F-automaton, we designed a technique to reassign the total order among the events having the same timestamp, namely timestamp-equivalent events.

Definition 4. [Timestamp-Equivalent Events] Given a log $\mathcal{L}$ and two events $e_{1}, e_{2} \in \mathcal{E}, e_{1}$ is timestamp-equivalent to $e_{2}$, i.e. $e_{1} \dot{\sim} e_{2}$, if and only if (iff) they belong to the same trace and they have the same timestamp. Formally, $e_{1} \dot{\sim} e_{2} \Leftrightarrow \mathcal{C}\left(e_{1}\right)=\mathcal{C}\left(e_{2}\right) \wedge \mathcal{T}\left(e_{1}\right)=\mathcal{T}\left(e_{2}\right)$. It is possible to identify the set of the timestamp-equivalent events as the quotient set of $\mathcal{E}$ by $\dot{\sim}$, formally, $\Omega \triangleq \mathcal{E} / \dot{\sim}$. 


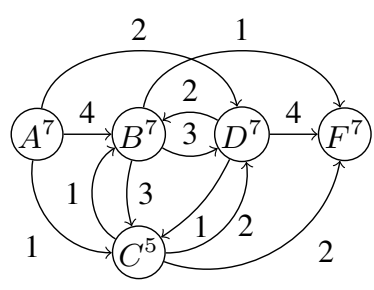

(a) L-Automaton.

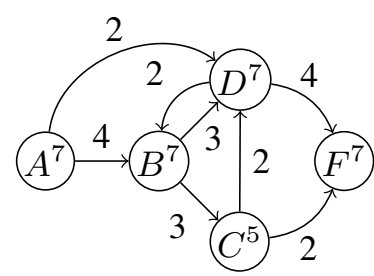

(b) F-Automaton.

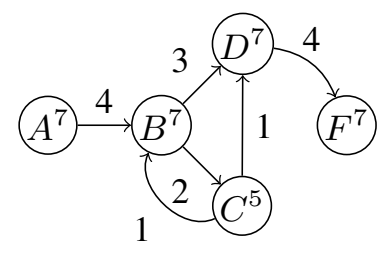

(c) RL-Automaton.

Fig. 1: Automata examples.

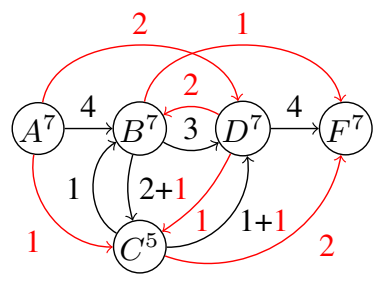

(a) L-Automaton.

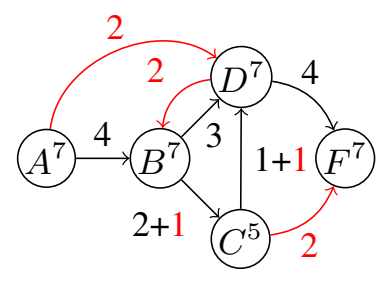

(b) F-Automaton.

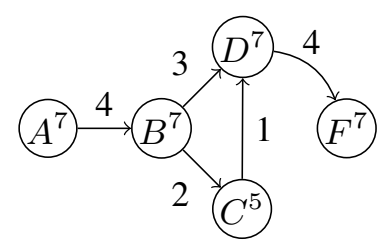

(c) RF-Automaton.

Fig. 2: Automata examples - UDFRs highlighted in red.

The presence of timestamp-equivalent events may lead to unreliable directly-follows relations (UDFRs). In the context of this paper, we say that a directly-follows relation, e.g. $d \rightsquigarrow b$, is unreliable iff it is derived from two timestamp-equivalent events, i.e. $d \dot{\sim} b$.

Definition 5. [Unreliable Directly-Follows Relation (UDFR)] Given two activities $x, y \in \Gamma$, there exists an unreliable directly-follows relation between $x$ and $y$, i.e. $x \rightsquigarrow u y$, iff $x \rightsquigarrow y \wedge$ $\exists e_{1}, e_{2} \in \mathcal{E} \mid \mathcal{A}\left(e_{1}\right)=x \wedge \mathcal{A}\left(e_{2}\right)=y \wedge e_{1} \dot{\sim} e_{2}$.

By construction, an L-automaton and even an F-automaton may include UDFRs. The $\log$ in Table 1 displays in red the timestamp-equivalent events. Projecting this information on the L-automaton and the F-automaton we can highlight the UDFRs, captured in red in Figures 2a and 2b.

It is possible to remove the UDFRs from both the L-automaton and the F-automaton. If we remove the UDFRs from the L-automaton, we refer to the resulting automaton as the refined L-automaton (RL-automaton, Figure 1c), while, if we remove the UDFRs from the F-automaton, we refer to the resulting automaton as the refined F-automaton (RF-automaton, Figure 2c). Given that techniques that remove infrequent directlyfollows relations are based on frequencies, in general, filtering the RL-automaton may not result into the RF-automaton. We rely on the RF-automata to repair timestampequivalent events.

Exploiting the frequency of a directly-follows relation, we introduce the function $\Phi: \Gamma \times \Gamma \rightarrow[0,1]$, which provides an estimate of the confidence level of a directlyfollows relation in an RF-automaton. This function is defined as: 


$$
\Phi(x, y) \triangleq \frac{\#(x, y)}{\sum_{z \in \Gamma} \#(x, z)}
$$

Then, we identify the optimal order of timestamp-equivalent events by selecting the most likely sequence of timestamp-equivalent events among all their possible sequences. Given a set of timestamp-equivalent events $\omega \in \mathcal{P}(\mathcal{E})^{2}$, first, we define the set of all possible sequences of timestamp-equivalent events in $\omega$ as:

$$
\mathcal{S}(\omega) \triangleq\left\{\bar{\omega} \in \mathcal{E}^{*}|| \bar{\omega}|=| \omega|\wedge \forall e \in \omega \exists 1 \leq i \leq| \omega \mid\left[e=\bar{\omega}_{i}\right]\right\}
$$

Then, using the confidence level of the directly-follows relations, we measure the confidence level of a sequence through the function $\%: \mathcal{E}^{*} \rightarrow[0,1]$. The latter function is ultimately used to discover the most likely sequence of a set of timestamp-equivalent events. Formally, given a set of timestamp-equivalent events $\omega \in \mathcal{P}(\mathcal{E})$, we refer to the sequence with the highest confidence level (i.e. the most likely sequence of a set of timestamp-equivalent events) as $\bar{\omega}^{o} \in \mathcal{S}(\omega)$, such that $\%\left(\bar{\omega}^{o}\right) \geq \%(\bar{\omega})[\forall \bar{\omega} \in \mathcal{S}(\omega)]$.

Following a pure probabilistic approach, the first implementation to come to mind for the function $\%: \mathcal{E}^{*} \rightarrow[0,1]$ is the following:

$$
\%(\bar{\omega}) \triangleq \prod_{i=1}^{|\bar{\omega}|-1} \Phi\left(\mathcal{A}\left(\bar{\omega}_{i}\right), \mathcal{A}\left(\bar{\omega}_{i+1}\right)\right)
$$

where the confidence level of the entire sequence is based on the likelihood of pairs of events following each other. While in theory this implementation works, in practice it may lead to a fault. Let us assume that we have three timestamp-equivalent events $e_{1}$, $e_{2}$, and $e_{3} \in \mathcal{E}$, with $\mathcal{A}\left(e_{1}\right)=a, \mathcal{A}\left(e_{2}\right)=b$, and $\mathcal{A}\left(e_{3}\right)=c$. Also, let us assume that only the following directly-follows relations hold: $a \rightsquigarrow b$ and $a \rightsquigarrow c$ (with a confidence level of 0.5 and 0.1 , respectively). Then, all the possible sequences that can be generated out of the three events will have a confidence level of 0 (since $b \rightsquigarrow c$ is missing), while, a sequence with $a \rightsquigarrow b$ should be preferred over other possible sequences (since $\Phi(a, b)$ is the highest). For example, we may consider the sequence $\langle a, b, c\rangle$ or $\langle c, a, b\rangle$ as the most likely.

Following this reasoning, an improved version of the function $\%: \mathcal{E}^{*} \rightarrow \mathbb{R}$ is:

$$
\%(\bar{\omega}) \triangleq \sum_{i=1}^{|\bar{\omega}|-1} \frac{\Phi\left(\mathcal{A}\left(\bar{\omega}_{i}\right), \mathcal{A}\left(\bar{\omega}_{i+1}\right)\right)}{|\bar{\omega}|-1}
$$

We note, however, that in the case of a set of events that are only observed as timestamp-equivalent, also this revised version of the function $\%$ would always return 0 . We acknowledge this as a potential limitation of our approach, since it is designed to repair the timestamp-equivalent events on the grounds of other observations of the same events with different timestamps.

Returning to our working example, the log in Table 1 contains three traces affected by timestamp-equivalent events (highlighted in red), specifically, $\bar{\omega}_{1}=\langle d, b, c\rangle, \bar{\omega}_{2}=$

\footnotetext{
${ }^{2}$ The powerset of $\mathcal{E}$.
} 


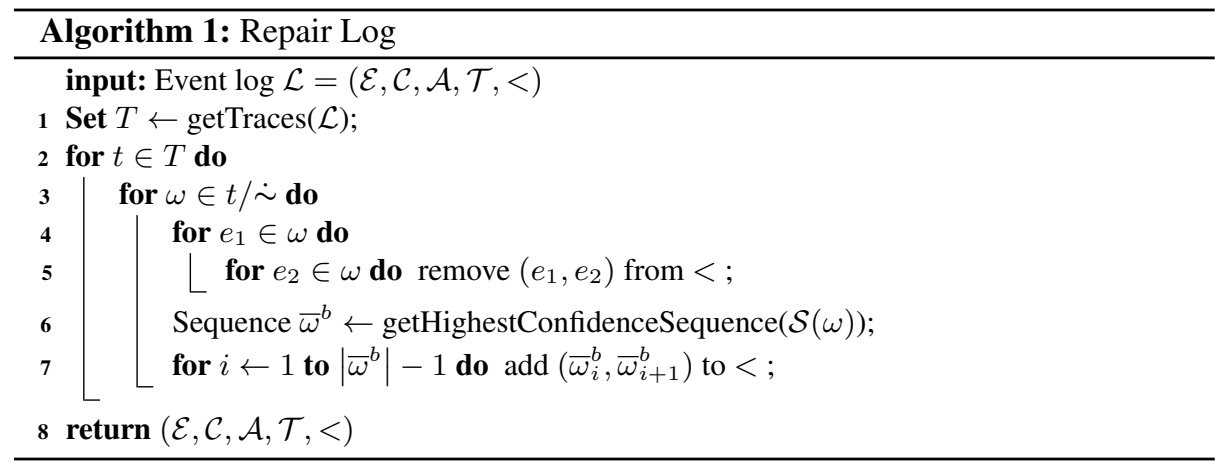

$\langle c, d, b\rangle$, and $\bar{\omega}_{3}=\langle c, d\rangle$. Our technique fixes each of the three sequences separately. Working on the RF-automaton (Figure 2c), we measure the confidence level of each possible sequence of the timestamp-equivalent events by analysing the confidence level of each arc of the RF-automaton. We recall that the confidence level of an arc, e.g. $\Phi(b, d)$, is equal to the number of times $(b, d)$ is traversed divided by the sum of the number of times each arc with source $b$ is traversed, i.e. $\Phi(b, d)=0.60$ as there are $2+3=5$ ways to leave node $b, 3$ of which are through the arc $(b, d)$.

Let us consider, $\bar{\omega}_{1}=\langle d, b, c\rangle$, Table 2 shows all the possible sequences and the respective confidence levels. We can see that the most likely sequence of events is $\bar{\omega}_{1}^{o}=\langle b, c, d\rangle$. Considering $\bar{\omega}_{2}=\langle c, d, b\rangle$, we would reach the same result, since the set of timestamp-equivalent events is the same as $\bar{\omega}_{1}$. While for the sequence $\bar{\omega}_{3}$, which contains only two events, we can trivially determine that $\bar{\omega}_{3}{ }^{o}=\langle d, c\rangle$, since $\Phi(c, d)=0$.

\begin{tabular}{c|c} 
Sequence $(\bar{\omega})$ & $\boldsymbol{\%}(\bar{\omega})$ \\
\hline$\langle D, B, C\rangle$ & $(\Phi(D, B)+\Phi(B, C)) / 2=0.2$ \\
$\langle D, C, B\rangle$ & $(\Phi(D, C)+\Phi(C, B)) / 2=0.0$ \\
$\langle B, C, D\rangle$ & $(\Phi(B, C)+\Phi(C, D)) / 2=0.7$ \\
$\langle B, D, C\rangle$ & $(\Phi(B, D)+\Phi(D, C)) / 2=0.3$ \\
$\langle C, B, D\rangle$ & $(\Phi(C, D)+\Phi(B, D)) / 2=0.3$ \\
$\langle C, D, B\rangle$ & $(\Phi(C, D)+\Phi(D, B)) / 2=0.5$ \\
\hline
\end{tabular}

Table 2: Selecting the sequence with the highest confidence level.

Algorithm 1 synthesises how our approach operates to repair a log affected by timestamp-equivalent events. For each trace $t$ in the log, we retrieve all the sets containing the timestamp-equivalent events belonging to that trace ( $\omega$, see line 2 and 3 ). Then, we perform two major operations over each set $\omega$. First, we remove the original strict total order defined for each pair of events $\left(e_{1}, e_{2}\right) \in \omega$ (lines 5). Second, we introduce the repaired total order based on the sequence of events with the highest confidence level (lines 6 and 7). After iterating this procedure for each trace in the log, we return the repaired log. 


\subsection{Time Complexity and ILP Formulation}

Time Complexity. The identification of the most likely sequence of events is an NPhard problem. We provide a sketch of its complexity by providing a polynomial time transformation from the Maximum Travelling Salesman Problem (Max TSP) (a well known NP-complete problem [17]) to the most likely sequence of events problem. The

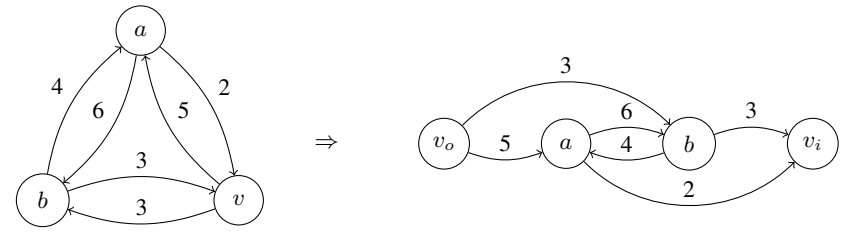

Fig. 3: Example, Max TSP reduction to most likely sequence of events problem.

Max TSP is the problem of identifying a Hamiltonian cycle (a tour that passes through all the vertices) with maximum cost in a complete asymmetric graph with non-negative weights. It is straightforward to show that a Max TSP problem can be reduced to an instance of the most likely sequence of events problem. This can be achieved through the application of the following six polynomial steps: (1) select a random vertex $v$ of the Max TSP problem; (2) introduce two new vertices $v_{i}$ and $v_{o}$; (3) for each incoming arc of $v$, i.e. $a=(s, v)$, create a new $\operatorname{arc} a_{i}=\left(s, v_{i}\right)$; (4) for each outgoing arc of $v$, i.e. $a=(v, t)$, create a new arc $a_{o}=\left(v_{o}, t\right)$; (5) remove all arcs connected to $v$; (6) remove vertex $v$. Now the Max TSP problem corresponds to determining the most likely sequence of events leading from $v_{o}$ to $v_{i}$. Figure 3 shows how to reduce a Max TSP problem to a most likely sequence of events problem. In the example, the asymmetric graph contains three vertices, $V=\{a, b, v\}$. These three vertices are connected via six weighted $\operatorname{arcs} A=\{(a, b, 6),(a, v, 2),(b, a, 4),(b, v, 3),(v, a, 5),(v, b, 3)\}$. As part of the reduction two new vertices $v_{i}$ and $v_{o}$ are introduced, as well as arcs connecting these vertices to vertices which were originally connected to $v$.

ILP Formulation. In section 3.2, we defined $\bar{\omega}^{o}$ as the most likely sequence over the set $\mathcal{S}(\omega)$ of possible sequences. To determine the most likely sequence over the set $\mathcal{S}(\omega)$ we propose the application of Integer Linear Programming (ILP), where the confidence level of a sequence is measured using the function proposed in equation 4 .

Given a $\log \mathcal{L}$, the inputs for our problem are the set of events $\omega \in \mathcal{E} / \dot{\sim}$, the event $s \in \mathcal{E}$ (not affected by timestamp error) which should precede the sequence $\bar{\omega}^{o}$, and the event $f \in \mathcal{E}$ (not affected by timestamp error) which should follow the sequence $\bar{\omega}^{o}$. Finally, for convenience we define the set $\omega^{f} \triangleq \omega \cup\{f\}$, and $\omega^{f s} \triangleq \omega \cup\{s, f\}$. Using $\omega^{f s}$ instead of $\omega$ allows us to take into account the context of the sequence $\omega$ when repairing the order of the events.

Before presenting the ILP formulation, we also need to introduce the following variables: i) for each event $e \in \omega^{f s}$ there exists an auxiliary variable $u_{e} \in \mathbb{Z} ;{ }^{3}$ ii) for each couple of events $\left(e_{1}, e_{2}\right) \in \omega^{f s} \times \omega^{f s}$ if $e_{1} \neq e_{2}$ there exists a variable

\footnotetext{
${ }^{3} u_{e}$ is a mathematical escamotage to ensure that the combination of equations will all have a strict order, which is defined by the ILP solver when it finds the solution.
} 
$x_{e_{1}, e_{2}} \in\{0,1\}$. If the solution of the ILP problem is such that $x_{e_{1}, e_{2}}=1$, event $e_{2}$ directly follow event $e_{1}$ in the most likely sequence.

The ILP problem aims at maximizing the confidence level of the sequence as proposed in equation 4 . We model the ILP problem of determining the best sequence as a traveling salesman problem (TSP) [18]. Our formulation presents two main differences from the original formulation. First, we try to maximize the distance instead of minimizing it (see Equation 5).

$$
\max \sum_{e_{1} \in \omega^{f s}} \sum_{e_{2} \in \omega^{f s}} \Phi\left(\mathcal{A}\left(e_{1}\right), \mathcal{A}\left(e_{2}\right)\right) \cdot x_{e_{1}, e_{2}} .
$$

Second, we impose that $f$ must close the cycle on $s$ (see Equation 6). The constraints of our ILP problem (Equation 5) are captured by Equation 6, 7, 8, and 9. ${ }^{4}$

$$
\begin{aligned}
& x_{f, s}=1 \quad \text { (6) } \quad \sum_{e_{2} \in \omega^{f s} \backslash\left\{e_{1}\right\}} x_{e_{1}, e_{2}}=1 \\
& \sum_{e_{2} \in \omega^{f s} \backslash\left\{e_{1}\right\}} x_{e_{2}, e_{1}}=1 \quad \text { (8) } u_{e_{1}}-u_{e_{2}}+\left|\omega^{f s}\right| \cdot x_{e_{1}, e_{2}} \leq\left|\omega^{f s}\right|-1
\end{aligned}
$$

In plain English they are the following. Equation 6; we connect the final event to the start event to prevent them from being in the middle of the sequence. Equation 7; we force each event $e_{1} \in \omega^{f}$ to precede one and only one event (Equation 7). Equation 8; we force each event $e_{1} \in \omega^{f}$ to follow one and only one event (Equation 8). Equation 9; for each couple of events $\left(e_{1}, e_{2}\right) \in \omega^{f} \times \omega^{f}$ where $e_{1} \neq e_{2}$, we prevent the presence of sub-tours in the TSP problem, i.e. we enforce the tour to visit all cities in one go (Equation 9). The latter, in our context, means that we enforce the presence of a single sequence covering all events.

\subsection{Event Timestamp Estimation}

Reordering timestamp-equivalent events does not fix their timestamps. Consequently, we complement our events-reordering approach with a technique to assign a timestamp to each reordered event.

To estimate an event timestamp, we rely on the distribution of the time elapsed between the event and the preceding event within the same trace. For simplicity, we refer to such time as duration of the event, whose distribution corresponds to its probability density function (PDF). Given an event $e \in \mathcal{E}$, we formally define its duration with the function $\mathcal{D}: \mathcal{E} \rightarrow \mathbb{N}$.

$$
\mathcal{D}(e) \triangleq \begin{cases}\mathcal{T}(e)-\mathcal{T}\left(e_{p}\right) & \text { if } \exists e_{p} \mid e_{p} \sqsubset e_{2} \wedge \mathcal{T}(e) \neq \mathcal{T}\left(e_{p}\right), \\ \epsilon & \text { otherwise }\end{cases}
$$

\footnotetext{
${ }^{4}$ Equation 7, 8, and 9 come from Miller et al. [18].
} 
Where $\epsilon$ is an arbitrary small value to avoid that when assigning a timestamp we will cause two events to be timestamp-equivalent.

Given activity $a \in \Gamma$, we can define the multiset of its events' durations as $\mathcal{D}_{a} \triangleq$ $\left\{(d, n) \in \mathbb{N} \times \mathbb{N}\left|\exists e \in \mathcal{E}_{a, d} \wedge n=\right| \mathcal{E}_{a, d} \mid\right\}$ where $\mathcal{E}_{a, d}$ is the subset of $\mathcal{E}$ containing only events of activity $a$ that have a given duration $d$, i.e. $\mathcal{E}_{a, d} \triangleq\{e \in \mathcal{E} \mid \mathcal{A}(e)=$ $a \wedge \mathcal{D}(e)=d\}$. We use the multiset to estimate the PDF of the duration of events of activity $a$. For this purpose we define the function estimating the duration of an event of activity $a$ as $f_{a}^{e s t}: \mathbb{N} \times \mathbb{N} \rightarrow(\mathbb{N} \rightarrow \mathbb{R})$, where the range is the PDF $\left(f_{a}\right)$ we want to estimate. This can be achieved using approaches as the one proposed by Silverman [19] for the estimation of multimodal distributions using kernel density.

Once the PDF of each activity is known, we can assign a new timestamp to each ordered event following the durations dictated by the PDF. The new timestamp is:

$$
\mathcal{T}^{+}(e) \triangleq \begin{cases}\mathcal{T}\left(e_{p}\right)+\overline{f_{\mathcal{A}(e)}} & \text { if } \exists e_{p} \mid e_{p} \sqsubset e_{2} \wedge \mathcal{T}(e) \neq \mathcal{T}\left(e_{p}\right) \\ \epsilon+\overline{f_{\mathcal{A}(e)}} & \text { otherwise }\end{cases}
$$

where $\overline{f_{\mathcal{A}(e)}}$ extracts a likely duration from the PDF $f_{\mathcal{A}(e)}$. Finally, the new timestamp for event $e$ substitutes the old one in the log, i.e. $\mathcal{T} \Leftarrow \mathcal{T} \oplus\left\{\left(e, \mathcal{T}^{+}(e)\right)\right\}$.

\section{Evaluation}

We implemented our approach as a standalone Java application, ${ }^{5}$ and used this prototype tool to evaluate the accuracy and time performance of our approach using artificial and real-life datasets. ${ }^{5}$ Finally, we conducted a case study to gain initial evidence on the usefulness of our approach in practice.

All the experiments reported in this section were performed on a 6-core Xeon E5$16503.5 \mathrm{Ghz}$ with $128 \mathrm{~GB}$ of RAM running JVM 8 with $48 \mathrm{~GB}$ of heap space.

\subsection{Datasets and Setup}

We generated the artificial dataset from the BPMN model shown in Figure 4. We used this model since it exhibits properties often found in models discovered from real-life logs, such as high degree of concurrency, unstructuredness, skips and loop structures. Starting from a reference log obtained by simulating this model, we injected different amounts of timestamp-equivalent events at three levels of granularity by changing the percentage of affected events, traces, or unique traces in increments of 5\%, ranging from $5 \%$ to $40 \%$. This produced three sets of eight logs, for a total of $24 \operatorname{logs}$. Each of the $24 \operatorname{logs}$ (as well as the reference $\log$ ) contains 3,000 traces, 45,330 events, and 24 activity labels. The logs' unique traces range from 1,391 to 2,361, while the reference $\log$ counts only 1,277 . Even though all the artificial logs were generated from a single artificial model as opposed to generating each log from different artificial models, we note that the validity of the assessment of our approach is grounded on the variety and amount of timestamp-equivalent events in each artificial log rather than on the variety of the behavior captured by the underlying model.

\footnotetext{
${ }^{5}$ Available at https://doi.org/10.6084/m9.figshare.11868969
} 


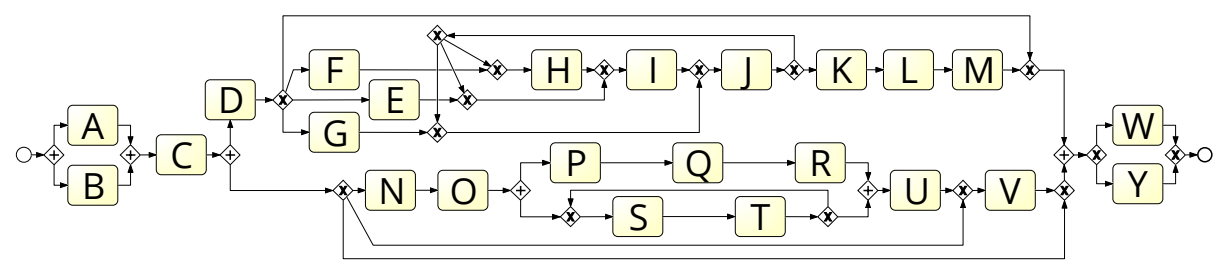

Fig. 4: Process model used to generate the artificial log

Alongside the artificial logs, we used the real-life log from the BPI Challenge 2014 [20]. We chose only this log out of the collection available at the 4TU Data Center $^{6}$ because it was the only one affected by timestamp-equivalent events for which we had access to the correct version (i.e. the original log without events' ordering errors), which we used as ground-truth in our experiments. The BPI2014 log counts 46,616 traces, 466,737 events, 39 activity labels, 22,632 unique traces. The $39 \%$ of its events are timestamp-equivalent, for a total of 182,027 , of which 113,334 are incorrectly ordered.

Using our approach we repaired the logs and measured the accuracy of the repair by applying the Levenshtein edit distance [21] between the proposed repair and the reference $\log$. We adapted the Levenshtein distance to the concept of traces, where instead of inserting, removing, or replacing characters we insert, remove, or replace events. We decided to use this metric since it provides a fine-grained measurement that allow us to assess the quality of a technique even in cases where the optimal solution is not identified. For the artificial logs, we also measured the fitness [22] of the repaired logs w.r.t. the BPMN model used as input, and the root-square mean error (RSME) between the assigned timestamp and the correct one.

We compared our approach against three baselines: no-repair, random repair, and naïve repair. No-repair simply does not apply any change to the affected $\log$, this baseline serves as bottom-line for improvement. Random repair randomly reorders the timestamp-equivalent events. Nä̈ve repair, for each set of timestampequivalent events, it collects all the

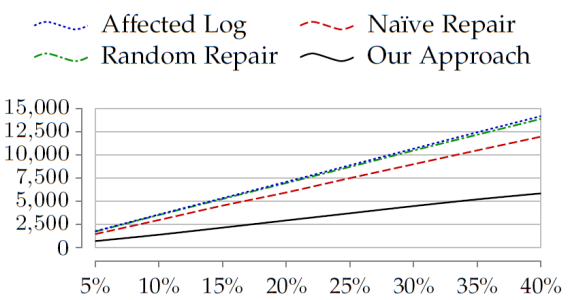

(a) Cumulative

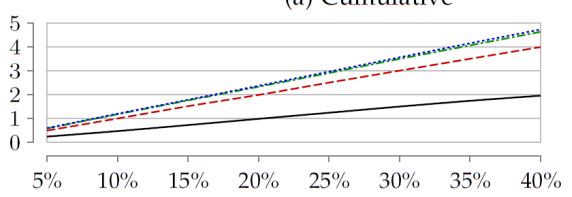

(b) Average over entire log

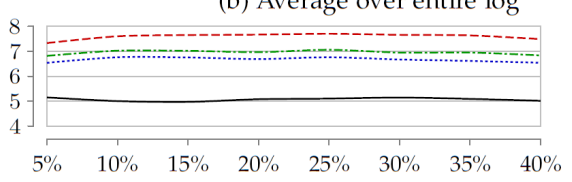

(c) Average over affected traces only

Fig. 5: Edit distance for the $\operatorname{logs} \mathrm{N} 5_{e}-\mathrm{N} 40_{e}$. traces containing such a set of events and it selects the most frequent trace out of them, then it reorders the specific set of timestamp-equivalent events according to the events' order in the most frequent trace.

${ }^{6}$ https://data.4tu.nl/repository/collection:event_logs_real 
We could not use any of the timestamp repair methods available in the literature $[6,8]$ because they address different types of timestamp errors, and both require as input a reference model annotated with execution times information.

\subsection{Results}

Tables 3 and 4 show the results of our evaluation. They report: the number of traces affected by timestamp-equivalent events, cumulative edit distance (cum.), maximum edit distance, average edit distance computed over the entire log and its standard deviation, average edit distance computed using only traces affected by timestamp-equivalent events and its standard deviation, RMSE and fitness (for artificial logs only).

Overall, the results show that our approach outperforms the three baselines in all the measurements, performing consistently better across the whole dataset, as captured by the values in bold in the tables. Figure 5 shows the plots of the cumulative edit distance, the average edit distance over the entire log, and the average edit distance over the affected traces only. Two things can be evinced from the graphs. First, the number of remaining timestamp-equivalent events is proportional to the number inserted, regardless of the approach we use. Second, our approach is the only one to always achieve an average edit distance on the affected traces that is below the one of the affected log (see Figure 5c). This implies that the random and naïve baselines are only repairing traces that are easy to repair, i.e. with an edit distance below the average. On the other hand, our approach can repair complex traces, in fact it reduced the average edit distance by $60 \%$ (on average across the dataset), and lowered the maximum edit distance by $12.5 \%$ (on average across the dataset).

Further, the results obtained when timestamp-equivalent events are injected at unique trace level are remarkable (see Table $4, \operatorname{logs} \mathrm{N} 5_{u t}-\mathrm{N} 40_{u t}$ ). In fact, the random and naïve baselines are not able to repair the log, i.e. they reduce the cumulative edit distance on average by $0 \%$ and $5 \%$ compared to the no-repair baseline, while our approach maintains an average improvement of $60 \%$. Figures $6 \mathrm{a}$ and $6 \mathrm{~b}$ provide a graphical snapshot of the benefits of our technique, as we can see, the curve representing our technique (black in colour) sits well below the baselines. While, the random and naïve baselines almost overlap with the no-repair baseline.

In terms of accuracy when assigning a timestamp to an event, we improve the RMSE on average by $54 \%$, with a minimum of $48 \%$ (achieved over

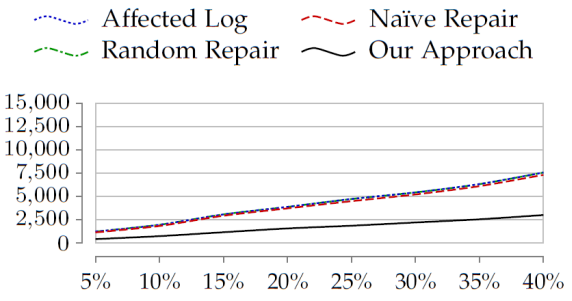

(a) Cumulative

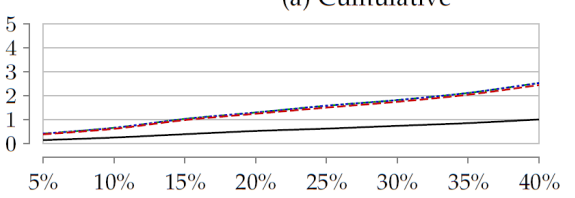

(b) Average over entire log

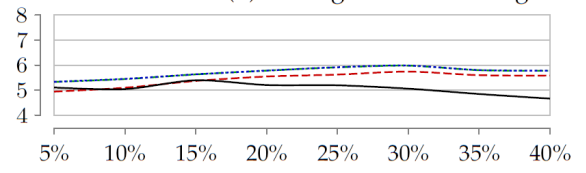

(c) Average over affected traces only

Fig. 6: Edit distance for the $\operatorname{logs} \mathrm{N} 5_{u t}-\mathrm{N} 40_{u t}$. 


\begin{tabular}{|c|c|c|c|c|c|c|c|c|c|c|}
\hline \multirow[b]{2}{*}{ Log } & \multirow[b]{2}{*}{ Approach } & \multirow[b]{2}{*}{$\begin{array}{c}\text { \#Affected } \\
\text { Traces }\end{array}$} & \multicolumn{2}{|c|}{ Distance } & \multicolumn{2}{|c|}{ Entire Log } & \multicolumn{2}{|c|}{ Affected Traces } & \multirow{2}{*}{$\begin{array}{c}\text { RMSE } \\
\text { in Days }\end{array}$} & \multirow[b]{2}{*}{ Fitness } \\
\hline & & & Cum. & Max & $\begin{array}{l}\text { Average } \\
\text { Distance }\end{array}$ & $\begin{array}{c}\text { Stand. } \\
\text { Dev. }\end{array}$ & \begin{tabular}{|l|} 
Average \\
Distance
\end{tabular} & $\begin{array}{c}\text { Stand. } \\
\text { Dev. }\end{array}$ & & \\
\hline \multirow{4}{*}{$\mathrm{N} 5{ }_{e}$} & No-repair & 273 & 1785 & 26 & 0.595 & 2.397 & 6.538 & 4.927 & 121.62 & 0.972 \\
\hline & & & 729 & 23 & 0.243 & 1.438 & & 4.318 & & 0.999 \\
\hline & Naïve & 202 & 1481 & 26 & 0.494 & 2.306 & 7.332 & 5.372 & 114.27 & 0.981 \\
\hline & Random & 249 & 1735 & 26 & 0.578 & 2.394 & 6.968 & 4.950 & 121.53 & 0.973 \\
\hline \multirow{4}{*}{$\mathrm{N} 10_{e}$} & No-repair & 531 & 3590 & 26 & 1.197 & 3.289 & 6.761 & 4.849 & 123.99 & 0.945 \\
\hline & Our & 286 & 1412 & 23 & 0.471 & 1.919 & 4.937 & 4.071 & 42.82 & 0.998 \\
\hline & Naïve & 394 & 2996 & 26 & 0.999 & 3.192 & 7.604 & 5.230 & 117.45 & 0.962 \\
\hline & Random & 504 & 3533 & 26 & 1.178 & 3.290 & 7.010 & 4.852 & 123.94 & 0.946 \\
\hline \multirow{4}{*}{$\mathrm{N} 15_{e}$} & No-repair & 792 & 5349 & 26 & 1.783 & 3.868 & 6.754 & 4.808 & 122.78 & 0.920 \\
\hline & & 444 & 2185 & 23 & 0.728 & 2.342 & 4.921 & .052 & 43.16 & 0.997 \\
\hline & Naïve & 594 & 4546 & 26 & 1.515 & 3.801 & & & 117.15 & 0.941 \\
\hline & Random & 747 & 5252 & 26 & 1.751 & 3.874 & & & & 0.922 \\
\hline & No-repair & 1062 & 7102 & 26 & 2.367 & 4.326 & & 4.897 & 123.58 & \\
\hline & & 586 & 2971 & 24 & 0.990 & 2.744 & & & 44.28 & .996 \\
\hline & Naï & 777 & 5960 & 26 & 1.987 & 4.290 & & & 117.77 & .924 \\
\hline & Rand & 997 & 6962 & 26 & 2.321 & 4.339 & 83 & 909 & 123.52 & 0.897 \\
\hline & No-repair & 1314 & 8885 & 26 & 2.962 & 4.668 & 6.762 & 4.904 & 123.98 & 0.867 \\
\hline & & 734 & 3742 & 24 & 1.247 & 3.027 & & & 44.83 & 0.995 \\
\hline & Naïve & 974 & 7504 & 26 & 2.501 & 4.680 & 7.704 & & 118.29 & 0.905 \\
\hline & Random & 1237 & 8723 & 26 & 2.908 & 4.689 & 52 & 909 & 123.94 & 0.871 \\
\hline & & & 10673 & 26 & & 4.898 & & & & 0.840 \\
\hline & & & & 24 & & & & & & \\
\hline & Naï & & & 26 & & & & & & 886 \\
\hline & & & 10490 & 26 & & 4.929 & & & 23.45 & .843 \\
\hline & No-1 & & 12440 & 26 & 4.147 & 5.042 & & .922 & 123.57 & 0.813 \\
\hline & & & 5226 & 24 & & & & & & .993 \\
\hline & Naï & & 10496 & 26 & 3.499 & 5.211 & & & .09 & 0.866 \\
\hline & Random & 1756 & 12185 & 26 & 4.062 & 5.093 & & & .54 & 0.818 \\
\hline & No-repair & & 14177 & 30 & .726 & 5.073 & & 74 & 23.34 & 0.785 \\
\hline & & 1174 & 5884 & 23 & & .542 & & & & 0.992 \\
\hline & Naïve & & 11969 & 30 & 3.9 & & & & & 0.847 \\
\hline & & & 13926 & 30 & & & & & & 0.791 \\
\hline & & & 995 & & & & & & & \\
\hline & & & & & & & & & & \\
\hline & & & 811 & 26 & & 1.748 & & & & 0.989 \\
\hline & Ran & & 971 & 26 & 0.324 & 1.822 & 6.986 & .014 & 120.62 & 0.985 \\
\hline & & & 1985 & 26 & & 2.511 & & 62 & 121.55 & 0.970 \\
\hline & & & 800 & 22 & & 1.485 & & & & 0.999 \\
\hline & & & 1647 & 26 & & & & & & 0.979 \\
\hline & & & 1951 & 26 & & 2.509 & & & & 0.970 \\
\hline & & & 2947 & & & 2.974 & & .746 & & 0.955 \\
\hline & & & & & & & & & & 0.998 \\
\hline & & & & & & & & & & 0.969 \\
\hline & & & 2901 & 26 & & 2.974 & & & & 0.956 \\
\hline & & & 043 & 26 & & 3.444 & & & 63 & 0.938 \\
\hline & & & 1584 & 23 & & 2.022 & & & & 0.998 \\
\hline & & & 3382 & 26 & & & & & 117.09 & 0.956 \\
\hline & & & 3960 & 26 & & 3.446 & & 4.790 & 123.60 & 0.939 \\
\hline & No-repair & 750 & 5082 & 26 & 1.694 & 3.799 & .776 & 4.826 & 123.44 & 0.924 \\
\hline & & & 2066 & 22 & & 2.295 & & & & 0.997 \\
\hline & & & 4318 & 26 & & & & & & 0.944 \\
\hline & & & 4990 & 26 & & 3.804 & & .821 & & 0.926 \\
\hline & No-re & & 6065 & & & 4.087 & & & & \\
\hline & & 48 & 2475 & 24 & & & & & & 0.996 \\
\hline & & & 105 & 26 & & 4.027 & & & & 0.934 \\
\hline & & & 5949 & 26 & & 4.096 & & 4.886 & 123.62 & 0.911 \\
\hline & No-re & 1050 & 7038 & 26 & 2.346 & 4.316 & 6.703 & 4.901 & 123.81 & 0.895 \\
\hline & & 578 & 2928 & 24 & & 2.727 & & 4.229 & & 0.996 \\
\hline & & & 5906 & 26 & 1.969 & 4.277 & & 5.248 & 117.97 & 0.925 \\
\hline & & & 6903 & 26 & & 4.329 & & 4.908 & 123.79 & 0.897 \\
\hline & & & & & & 4.504 & & & 123.90 & 0.880 \\
\hline & & & 3317 & 2 & & 2.868 & & 4.202 & & 0.995 \\
\hline & & & 6741 & 26 & & 4.492 & & 5.230 & 118.06 & 0.914 \\
\hline & Random & 1132 & 7891 & 26 & 2.630 & 4.521 & 6.971 & 4.889 & 123.87 & 0.882 \\
\hline
\end{tabular}

Table 3: Results of the experimental evaluation - Part 1. 


\begin{tabular}{|c|c|c|c|c|c|c|c|c|c|c|}
\hline \multirow[b]{2}{*}{ Log } & \multirow[b]{2}{*}{ Approach } & \multirow[b]{2}{*}{$\begin{array}{c}\text { \#Affected } \\
\text { Traces }\end{array}$} & \multicolumn{2}{|c|}{ Distance } & \multicolumn{2}{|c|}{ Entire Log } & \multicolumn{2}{|c|}{ Affected Traces } & \multirow[b]{2}{*}{$\begin{array}{l}\text { RMSE } \\
\text { in Days }\end{array}$} & \multirow[b]{2}{*}{ Fitness } \\
\hline & & & Cum. & $\operatorname{Max}$ & $\begin{array}{l}\text { Average } \\
\text { Distance }\end{array}$ & $\begin{array}{c}\text { Stand. } \\
\text { Dev. }\end{array}$ & $\begin{array}{l}\text { Average } \\
\text { Distance }\end{array}$ & $\begin{array}{c}\text { Stand. } \\
\text { Dev. }\end{array}$ & & \\
\hline \multirow{4}{*}{$\mathrm{N} 5_{u t}$} & No-repair & 235 & 1253 & 22 & 0.418 & \begin{tabular}{|l|}
1.878 \\
\end{tabular} & 5.332 & 4.340 & 109.84 & 0.978 \\
\hline & Our & 87 & 444 & 16 & 0.148 & 1.077 & 5.103 & 3.833 & 55.71 & 0.999 \\
\hline & Naïve & 235 & 1160 & 22 & 0.387 & 1.821 & 4.936 & 4.457 & 104.74 & 0.984 \\
\hline & Random & 235 & 1252 & 22 & 0.417 & 1.878 & 5.328 & 4.343 & 109.82 & 0.978 \\
\hline \multirow{4}{*}{$\mathrm{N} 10_{u t}$} & No-repair & 363 & 1978 & 25 & 0.659 & 2.377 & 5.449 & 4.539 & 114.36 & 0.967 \\
\hline & Our & 151 & 761 & 19 & 0.254 & 1.402 & 5.040 & 3.865 & 58.06 & 0.999 \\
\hline & Naïve & 363 & 1849 & 25 & 0.616 & 2.319 & 5.094 & 4.652 & 110.99 & 0.974 \\
\hline & Random & 363 & 1975 & 25 & 0.658 & 2.376 & 5.441 & 4.544 & 114.34 & 0.968 \\
\hline \multirow{4}{*}{$\mathrm{N} 15_{u t}$} & No-repair & 549 & 3092 & 25 & 1.031 & 2.956 & 5.632 & 4.672 & 114.92 & 0.949 \\
\hline & Our & 219 & 1181 & 19 & 0.394 & 1.777 & 5.393 & 4.039 & 57.98 & 0.999 \\
\hline & Naïve & 549 & 2946 & 25 & 0.982 & 2.911 & 5.366 & 4.773 & 112.68 & 0.958 \\
\hline & Random & 549 & 3089 & 25 & 1.030 & 2.955 & 5.627 & 4.676 & 114.90 & 0.950 \\
\hline \multirow{4}{*}{$\mathrm{N} 20_{u t}$} & No-repair & 674 & 3895 & 25 & 1.298 & 3.288 & 5.779 & 4.715 & 116.26 & 0.950 \\
\hline & Our & 305 & 1587 & 21 & 0.529 & 2.028 & 5.203 & 4.016 & 59.31 & 0.998 \\
\hline & Naïve & 674 & 3736 & 25 & 1.245 & 3.250 & 5.543 & 4.816 & 114.44 & 0.949 \\
\hline & Random & 674 & 3895 & 25 & 1.298 & 3.288 & 5.779 & 4.715 & 116.23 & 0.939 \\
\hline \multirow{4}{*}{$\mathrm{N} 25_{u t}$} & No-repair & 802 & 4745 & 25 & 1.582 & 3.565 & 5.916 & 4.680 & 116.36 & 0.925 \\
\hline & Our & 362 & 1879 & 19 & 0.626 & 2.160 & 5.191 & 3.871 & 60.14 & 0.998 \\
\hline & Naïve & 802 & 4507 & 25 & 1.502 & 3.511 & 5.620 & 4.792 & 113.70 & 0.938 \\
\hline & Random & 802 & 4739 & 25 & 1.580 & 3.564 & 5.909 & 4.684 & 116.34 & 0.926 \\
\hline \multirow{4}{*}{$\mathrm{N} 30_{u t}$} & No-repair & 911 & 5449 & 25 & 1.816 & 3.764 & 5.981 & 4.663 & 116.51 & 0.915 \\
\hline & Our & 440 & 2225 & 19 & 0.742 & 2.330 & 5.057 & 3.897 & 59.83 & 0.997 \\
\hline & Naïve & 911 & 5228 & 25 & 1.743 & 3.722 & 5.739 & 4.763 & 114.48 & 0.928 \\
\hline & Random & 911 & 5446 & 25 & 1.815 & 3.764 & 5.978 & 4.665 & 116.49 & 0.916 \\
\hline \multirow{4}{*}{$\mathrm{N} 35_{u t}$} & No-repair & 1093 & 6341 & 25 & 2.114 & 3.949 & 5.801 & 4.626 & 115.02 & 0.902 \\
\hline & Our & 530 & 2567 & 20 & 0.856 & 2.450 & 4.843 & 3.829 & 59.63 & 0.997 \\
\hline & Naïve & 1093 & 6117 & 25 & 2.039 & 3.914 & 5.597 & 4.704 & 113.24 & 0.916 \\
\hline & Random & 1093 & 6335 & 25 & 2.112 & 3.948 & 5.796 & 4.629 & 115.00 & 0.903 \\
\hline \multirow{4}{*}{$\mathrm{N} 40_{u t}$} & No-repair & 1313 & 7584 & 25 & 2.528 & 4.134 & 5.776 & 4.505 & 114.83 & 0.882 \\
\hline & Our & 649 & 3023 & 19 & 1.008 & 2.575 & 4.658 & 3.695 & 60.13 & 0.996 \\
\hline & Naïve & 1313 & 7330 & 25 & 2.443 & 4.100 & 5.583 & 4.569 & 112.86 & 0.895 \\
\hline & Random & 1313 & 7582 & 25 & 2.527 & 4.134 & 5.775 & 4.506 & 114.82 & 0.882 \\
\hline \multirow{4}{*}{ BPI2014 } & No-repair & 36442 & 144957 & 63 & 3.110 & 3.445 & 3.978 & 3.425 & - & - \\
\hline & Our & 19838 & 77312 & 63 & 1.658 & 2.968 & 3.897 & 3.461 & - & - \\
\hline & Naïve & 35000 & 142072 & 63 & 3.048 & 3.482 & 4.059 & 3.471 & - & - \\
\hline & Random & 34070 & 140199 & 63 & 3.008 & 3.505 & 4.115 & 3.501 & - & - \\
\hline
\end{tabular}

Table 4: Results of the experimental evaluation - Part 2.

the most complex log: $\mathrm{N} 40_{u t}$ ) and a maximum of 59\%. Additionally, it should be noted that the random and naïve baselines only achieve on average an improvement of $0 \%$ and $4 \%$ (respectively). Moreover, the fitness of the logs repaired by our approach is the highest across all tests.

Finally, the results on the real-life log support the fact that our approach noticeably outperforms the three baselines. Indeed, while the random repair and the naïve repair improve the cumulative edit distance of $3 \%$ and $2 \%$ (resp.) compared to the no-repair baseline, our approach brings an improvement of $47 \%$. These results, alongside those obtained on the artificial logs, confirm the efficacy of our approach.

Time Performance. On the artificial logs, our event-reordering technique achieved an average execution time of $4.9 \mathrm{~s}$ ( $\min : 0.9 \mathrm{~s}$, max: $20.2 \mathrm{~s}$ ), linearly increasing with the number of timestamp-equivalent events. The time required to repair the BPI2014 log was over $45 \mathrm{~m}$, due to the high number of affected events (over 180,000), with an average time to fix a single event of $15 \mathrm{~ms}$. Overall, the bulk of the time is taken by our timestamp estimation technique, with an average of $4.7 \mathrm{~m}$ on the artificial logs (min: $1.8 \mathrm{~m}$, max: $24.4 \mathrm{~m}$ ), and $5.4 \mathrm{~m}$ for the BPI2014 log. Such performance is expected, due 
to the inherent complexity of estimating the probability density function (via kernel estimation) associated with the duration of each event.

\subsection{Case Study}

In collaboration with an Australian consultancy company we applied our approach to an event log from the meat $\&$ livestock domain. The process recorded in the log covers all stages livestock goes through from when cows are bred or purchased at the market, to when they are slaughtered and the meat is processed. The event log contains data originating from four different cattle farms (for a total of 18,192 events), and records the processing of 3,032 cows over a timeframe of about two years. In this context, thus, each animal identifies a process case. Each cow is RFID-tagged, to record the activity each cow is involved in (e.g. breading, backgrounding, feeding) throughout the various stages of this process.

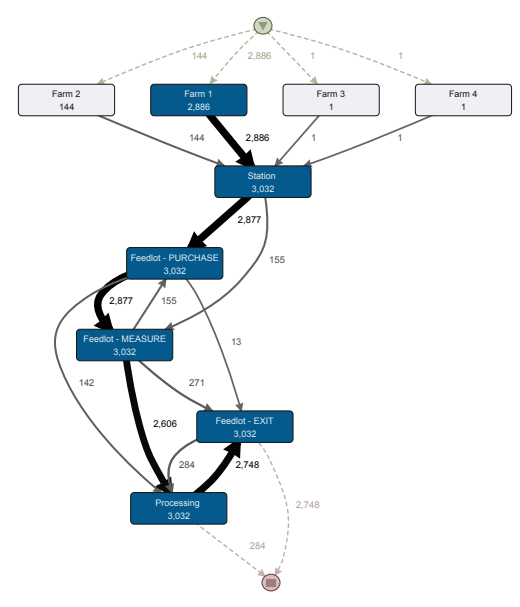

(a) Original

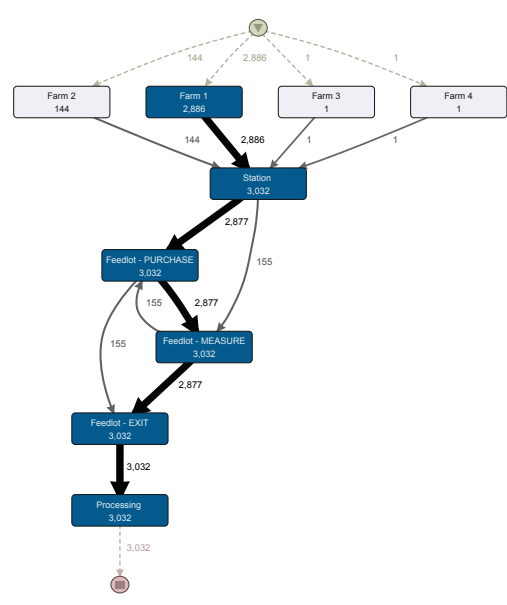

(b) Repaired

Fig. 7: Directly-follows graph of the log before and after repairing (shown in Disco).

When analysing the event log, we observed it contained 5,496 timestamp-equivalent events out of 18,192. Specifically, the timestamp of these events did not capture the time when the event occurred (i.e. hours and minutes) but only the date. As already mentioned, this type of problem is frequent in real-life event logs, and depends on the granularity of the logging system. In our case study, this resulted in 2,748 cases where cows were slaughtered (activity "Processing") before leaving the feeding lot (activity "Feedlot - EXIT"), as can be observed in Figure 7a. This transition is not possible in reality, as confirmed by domain experts.

The application of our approach required no domain knowledge or additional processing of the log. Figure $7 \mathrm{~b}$ shows the directly-follows graph of the log after repair, 
where the order of activities "Feedlot - EXIT" and "Processing" has been swapped. Our approach was able to repair all inaccurate cases, requiring on average $66.5 \pm 3.08^{7}$ sec to repair the log. The resulting graph is less cluttered and thus more readable. For example, the process cases that inaccurately transitioned from "Feedlot - PURCHASE" to "Processing" have now converged into the edge going from "Feedlot - PURCHASE" to "Feedlot - EXIT". This graph was validated by the business analyst involved in the case study, who confirmed the correct sequencing of activities.

\section{Conclusion}

We proposed an automated approach for correcting same-timestamp errors in event logs. These errors are quite common in real-life event logs [2,3,1], and may lead to quality issues in process mining analysis. The approach first detects the correct order of the events having the same timestamp, and then assigns a timestamp to each reordered event relying on a multimodal distribution of the duration of process activities.

We implemented our approach and assessed its effectiveness and efficiency via an experimental evaluation with artificial and real-life logs, and a case study. The experiments show that our approach reduces the amount of incorrect timestamps by at least $50 \%$, both on artificial and real-life logs, while, the case study conducted in the meat $\&$ livestock domain provides initial results on the usefulness of our approach in practice. However, more experiments in real-life settings need to be conducted to obtain confirmatory evidence.

A possible avenue for future work is to fix events erroneously recorded with incorrect (different) timestamps by humans. This scenario occurs when the corresponding activities are performed one shortly after the other. For example, this has been observed for activities performed just after midnight in healthcare logs, where the date is incorrectly recorded as that of the previous day, while the time is recorded correctly. This issue of data quality is known as the "inadvertent time travel" pattern [1]. Another human error leading to this pattern is when the user inadvertently presses keys adjacent to the intended ones when entering an event timestamp into a log [1]. An idea would be to adapt our approach by introducing a preliminary step that, using our probabilistic model, identifies such cases and assigns them an equal timestamp. This would allow us to treat them by applying the approach proposed in this paper.

Lastly, another interesting avenue for future work is to extend the current approach to repair event logs with same-timestamp errors in the presence of multiple life-cycle events for each activity. Specifically, if there are only start and complete events for each activity, we can process all the start events and all the complete events separately, fixing the two types of event in two stages. On the other hand, the presence of multiple life-cycle events per activity would warrant a more elaborated approach.

Acknowledgments This research is partly funded by the Australian Research Council (DP180102839).

\footnotetext{
${ }^{7}$ Over a set of five executions.
} 


\section{References}

1. S. Suriadi, R. Andrews, A. ter Hofstede, and M. Wynn, "Event log imperfection patterns for process mining: Towards a systematic approach to cleaning event logs," Information Systems, vol. 64, pp. 132-150, 2017.

2. R. Bose, R. Mans, and W. van der Aalst, "Wanna improve process mining results?," in 2013 IEEE (CIDM), pp. 127-134, IEEE, 2013.

3. R. Mans, W. van der Aalst, R. Vanwersch, and A. Moleman, "Process mining in healthcare: Data challenges when answering frequently posed questions," in Process Support and Knowledge Representation in Health Care, Springer, 2012.

4. L. Ghionna, G. Greco, A. Guzzo, and L. Pontieri, "Outlier detection techniques for process mining applications," in ISMIS, pp. 150-159, Springer, 2008.

5. R. Conforti, M. La Rosa, and A. ter Hofstede, "Filtering out infrequent behavior from business process event logs," IEEE TKDE, vol. 29, no. 2, pp. 300-314, 2016.

6. A. Rogge-Solti, R. Mans, W. van der Aalst, and M. Weske, "Improving documentation by repairing event logs," in IFIP Working Conference on The Practice of Enterprise Modeling, pp. 129-144, Springer, 2013.

7. J. Wang, S. Song, X. Lin, X. Zhu, and J. Pei, "Cleaning structured event logs: A graph repair approach," in Proceedings of IEEE ICDE, pp. 30-41, IEEE, 2015.

8. S. Song, Y. Cao, and J. Wang, "Cleaning timestamps with temporal constraints," VLDB Endowment, vol. 9, no. 10, pp. 708-719, 2016.

9. M. Sani, S. van Zelst, and W. van der Aalst, "Improving Process Discovery Results by Filtering Outliers using Conditional Behavioural Probabilities," in International Workshop on Business Process Intelligence (BPI'17), 2017.

10. M. Sani, S. van Zelst, and W. van der Aalst, "Repairing outlier behaviour in event logs using contextual behaviour," EMISAJ, vol. 14, pp. 1-24, 2019.

11. S. van Zelst, M. Fani Sani, A. Ostovar, R. Conforti, and M. La Rosa, "Filtering spurious events from event streams of business processes," in (CAiSE), Springer, 2018.

12. S. van Zelst, M. Fani Sani, A. Ostovar, R. Conforti, and M. La Rosa, "Detection and removal of infrequent behaviour from event streams of business processes," Information Systems, 2019.

13. W. van der Aalst, Process Mining: Data Science in Action. Springer, 2016.

14. M. Dumas, M. La Rosa, J. Mendling, and H. Reijers, Fundamentals of Business Process Management. Springer, 2nd ed., 2018.

15. S. Leemans, D. Fahland, and W. van der Aalst, "Discovering block-structured process models from event logs containing infrequent behaviour," in BPM Workshops, Springer, 2014.

16. A. Augusto, R. Conforti, M. Dumas, M. La Rosa, and A. Polyvyanyy, "Split miner: automated discovery of accurate and simple business process models from event logs," Knowledge and Information Systems, vol. 59, no. 2, pp. 251-284, 2019.

17. M. Lewenstein and M. Sviridenko, "Approximating asymmetric maximum TSP," in SODA, Society for Industrial and Applied Mathematics, 2003.

18. C. Miller, A. Tucker, and R. Zemlin, "Integer programming formulation of traveling salesman problems," Journal of the ACM, vol. 7, no. 4, pp. 326-329, 1960.

19. B. Silverman, "Using kernel density estimates to investigate multimodality," Journal of the Royal Statistical Society. Series B (Methodological), pp. 97-99, 1981.

20. B. van Dongen, "BPI Challenge 2014: Activity log for incidents," 2014.

21. V. I. Levenshtein, "Binary codes capable of correcting deletions, insertions and reversals," in Soviet physics doklady, vol. 10, p. 707, 1966.

22. A. Adriansyah, B. van Dongen, and W. van der Aalst, "Conformance checking using costbased fitness analysis,” in EDOC, pp. 55-64, IEEE, 2011. 